\title{
The anaesthetic management of a compromised airway secondary to lemierre's syndrome
}

\begin{abstract}
Lemierre's syndrome is a life threatening soft tissue infection of the neck. We describe the anaesthetic management of a patient presenting with a compromised airway due to severe soft tissue swelling associated with Lemierre's syndrome. In this case, preoperative computed tomography and nasal endoscopy delineated the anatomy. The otolaryngology team were prepared for an airway emergency. High flow humidified nasal oxygen maximised oxygenation during awake fibreoptic intubation and the time available for rescue tracheostomy if required.
\end{abstract}

Keywords: anaesthesia, awake fibreoptic intubation, lemierre's syndrome
Volume 9 Issue 3 - 2017

\author{
D Levy,' K Flower,' T Hinde,' T Ali,' CVinall,', \\ A Varvinskiy' \\ 'Department of Anaesthesia and Perioperative Medicine, Torbay \\ Hospital,Torquay, United Kingdom \\ ${ }^{2}$ Department of Oral and Maxillofacial surgery, Royal Devon and \\ Exeter Hospital, Exeter United Kingdom
}

Correspondence: David Levy, Department of Anaesthesia and Perioperative Medicine, Torbay Hospital, Lowes Bridge, Torquay, United Kingdom, Tel 018036I4567, Email d.levyl@nhs.net

Received: October 12, 2017 | Published: December II, 2017
Abbreviations: HFNO, high flow humidified nasal oxygen; AFOI, awake fibreoptic intubation

\section{Introduction}

Lemierre's syndrome describes a severe soft tissue infection of the head and neck leading to internal jugular vein thrombosis and septic emboli, most commonly to the lungs. It typically occurs following infections of the tonsils or pharynx, although tooth extraction is a rare but recognised caus. ${ }^{1,2}$ The most common causative organism is the gram-negative anaerobic bacterium Fusobacterium necrophorum. ${ }^{3}$ Prior to the development of antibiotics, mortality was said to be as high as $90 \%$ but following their introduction, the incidence of Lemierre's syndrome has fallen so low that it has been referred to as the "forgotten disease" ${ }^{3,4}$ A more recent increase in the number of documented cases may indicate a rise in incidence, possibly due to antibiotic resistance and changes in prescription patterns ${ }^{2}$. The majority of those affected are previously health children and young adults. ${ }^{4}$ It most commonly presents with sore throat, fever, dysphagia, trismus, neck mass and pain, potentially making airway management extremely challenging. ${ }^{2,3}$ Other symptoms may include pleuritic chest pain, dyspnoea and haemoptysis. ${ }^{4}$ Management should focus on surgical drainage of abscess in combination with targeted antibiotic therapy, while the role of anticoagulation for venous thrombosis remains controversial. ${ }^{2}$

A variety of methods have been described in the literature for airway management in the presence of soft tissue infections of the neck; including intravenous and gas inductions, direct laryngoscopy, blind nasal intubation, asleep and awake fibreoptic intubation and awake tracheostomy. ${ }^{5}$ Case reports exist describing the use of cervical plexus blocks to allow incision and drainage, avoiding instrumentation of the airway. ${ }^{6}$ We present the case of an immunosuppressed patient requiring emergency incision and drainage of a large neck abscess, and the steps we took to minimise the risk associated with endotracheal intubation.

\section{Case presentation}

A 67 year old lady presented to Torbay Hospital, a district general hospital, three days following the extraction of her lower right wisdom tooth with swelling and pain of her right jaw and neck, swelling of her tongue and difficulty swallowing. Medical history included rheumatoid arthritis managed with hydroxychloroquine and methotrexate. Examination revealed significant submandibular swelling extending to the submental region, and erythema extending to her sternal notch. Mouth opening was reduced to $15 \mathrm{~mm}$, Mallampati score was 3 and neck extension was significantly limited due to the sizeable right sided neck swelling and pain and her underlying rheumatoid arthritis. In spite of these findings, she was physiologically more stable than expected. Computed tomography of the neck demonstrated a right submandibular, buccal and sublingual collection extending to the right parapharyngeal, carotid, retropharyngeal and prevertebral spaces. This had resulted in displacement of the oropharynx to the left and narrowing of the airway superiorly. Infection had extended so far as to cause apical lung abscesses and had led to a right internal jugular thrombosis.

Appropriate antibiotics and a course of dexamethasone were commenced. A fibreoptic nasendoscopy performed by the otolaryngology team demonstrated right parapharyngeal swelling and an oedematous right arytenoid, but importantly showed mobile vocal cords and suggested fibreoptic intubation would be possible. The tertiary centre cardiothoracics team reviewed the images and felt a thoracoscopic washout was not necessary.

Following administration of glycopyrrolate as an anti-sialagogue, High Flow Humidified Nasal Oxygen (HFNO: Optiflow ${ }^{\mathrm{TM}}$ Fisher and Paykel) was commenced with remifentanil sedation. The nasal and oral muscosa were anaesthetised with direct sprays of $4 \%$ lidocaine and the airway was anaesthetised with $1 \mathrm{ml}$ aliquots of $4 \%$ lidocaine plus $9 \mathrm{ml}$ of air ejected from the working channel of fibreoptic scope. Awake fibreoptic intubation (AFOI) demonstrated significant distortion of the anatomy but was well tolerated with oxygen saturations maintained throughout and a cuffed nasal endotracheal tube was passed on the first attempt without issue. Following induction of general anaesthesia copious pus was irrigated from the sublingual, submandibular and parapharyngeal spaces.

The patient was admitted to intensive care and electively extubated the next day after demonstration of a cuff leak and further fibreoptic examination by the otolaryngology team. Following successful 
extubation, the patient was transferred to the ward to continue intravenous antibiotics and observation. The patient was discharged six days later with oral antibiotics and cessation of immunosuppression medication until follow up in six weeks by the respiratory team regarding the apical lung abscesses (Figure 1).

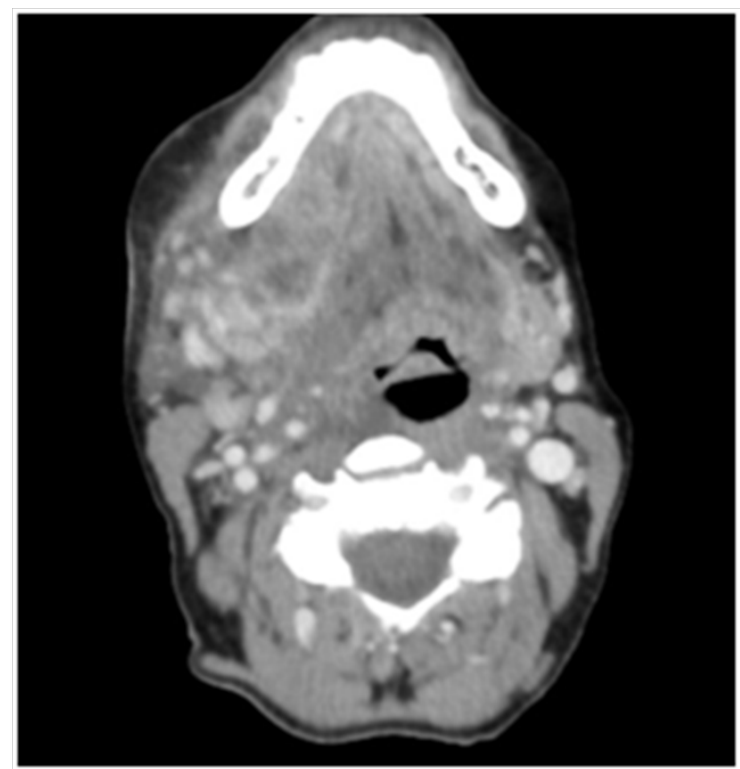

Figure I Axial slice computed tomography demonstrating a large collection displacing the oropharynx to the left.

\section{Discussion}

Soft tissue infections of the neck present a multitude of problems for the anaesthetist. This patient group may be haemodynamically compromised as a result of sepsis. Airway swelling can make visualisation of the anatomy extremely difficult. Induction prior to securing of the airway risks airway collapse and an inability to ventilate. The potential for infection to progress rapidly can preclude deferring for more experienced hands. Swelling can be so significant as to occlude PVC tracheal tubes. ${ }^{7}$ Traumatic intubation may release pus, obscure the view and potentially lead to contamination of the bronchial tree. ${ }^{8}$ Anterior spread of infection can make surgical tracheostomy difficult due to the surrounding induration and is a relative contraindication due to the risk of mediastinal spread. ${ }^{8,9}$

In this case we anticipated that that face-mask ventilation and direct laryngoscopy might be difficult or impossible. Features of particular concern in this patent were trismus, dysphagia, poor Mallampati grade, reduced neck extension and abscess of the neck with radiological evidence of compression and distortion of the airway. It is possible that induction of general anaesthesia prior to securing the airway could have caused complete airway obstruction with the relaxation of the pharyngeal muscles. We were also concerned that efforts at direct laryngoscopy may lead to traumatic rupture of the abscess causing contamination of the airway. It was our assessment that AFOI was the safest option available for airway management to allow surgical drainage of the sizeable abscess.

Appropriate preparation included the presence of an experienced anaesthesiologist and assistant and consideration of alternative airway plans, thus ensuring that the otolaryngology team were aware and prepared for an airway emergency. A multidisciplinary approach established that the case was conducted in the appropriate facility. Preoperative nasendoscopy allowed anatomical assessment to discern whether AFOI was likely to succeed. HFNO has previously been demonstrated to be well tolerated in spontaneously breathing patients undergoing AFOI and may potentially prevent desaturation arising as a result of apnoea or hypoventilation. ${ }^{10}$ In this case it did not hinder AFOI, maximising oxygenation and the time available for rescue tracheostomy if it were to be required. AFOI proved to be a good option as it was well tolerated allowed good visualisation of the anatomy, and minimised trauma.

We concluded that long-term immunosuppressants may have masked the significant inflammatory response expected in this case, highlighting how initial appearances may be deceptive. It is possible that had we not intervened in a timely manner, the airway compromise may have progressed further making endotracheal intubation unlikely to succeed. Challenges included the co-ordination of diagnosis and planning between five clinical teams over two sites. This meant that time critical source control occurred late in the evening with potentially fewer resources available.

Odontogenic infection is an uncommon precipitant of Lemierre's syndrome, a rare disease with an apparently increasing incidence. ${ }^{1}$ With the potential to present as an airway emergency requiring urgent drainage, it is a disease that anaesthesiologist and critical care physicians should be familiar with.

\section{Acknowledgments}

None.

\section{Conflicts of interest}

The authors declare no conflicts of interest.

\section{References}

1. Ghaly B, Bertram A, Naim A. Lemierre's syndrome: a serious complication of a routine dental procedure. Aust Dent J. 2013;58(2):246249

2. Karkos PD, Asrani S, Karkos CD, et al. Lemierre's syndrome: $\mathrm{A}$ systematic review. Laryngoscope. 2009;119(8):1552-1559.

3. Eilbert W, Singla N. Lemierre's syndrome. Int $J$ Emerg Med. 2013;6(1):40.

4. Noy D, Rachmiel A, Levy-Faber D, et al. Lemierre's syndrome from odontogenic infection: Review of the literature and case description. Ann Maxillofac Surg. 2015;5(2):219-225.

5. Loughnan TE, Allen DE. Ludwig's angina. The anaesthetic management of nine cases. Anaesthesia. 1985;40(3):295-297.

6. Manish M, Mehrotra S. Decompression of Ludwig's angina under cervical block. Anesthesiology. 2002;97(6):1625-1626.

7. Chung RA, Liban JB. Ludwig's angina and tracheal tube obstruction. Anaesthesia. 1991;46: 228-239.

8. Neff SPW, Merry AF, Anderson B. Airway Management in Ludwig's Angina. Anaesth Intensive Care. 1999;27(6):659-661.

9. Sujatha MP, Madhusudhana R, Amrutha KS, et al. Anaesthetic management of Ludwig's angina with comorbidities. Indian J Anaesth. 2015;59(10):679-681.

10. Badiger S, John M, Fearnley RA, et al. Optimizing oxygenation and intubation conditions during awake fibre-optic intubation using a highflow nasal oxygen-delivery system. Br J Anaesth. 2015;115(4):629-632. 